\title{
COEFFICIENTS FOR ALPHA-CONVEX UNIVALENT FUNCTIONS
}

\author{
BY P. K. KULSHRESTHA
}

Communicated by Eugene Isaacson, September 29, 1973

Let $\alpha$ be a nonnegative real number, and let $M(\alpha)$ denote the class of normalized $\alpha$-convex univalent functions $f$ in the open unit disc $E=\{z:|z|<1\}$, i.e., $f \in M(\alpha)$ if and only if $f$ is regular in $E, f(0)=f^{\prime}(0)-$ $1=0, f(z) f^{\prime}(z) / z \neq 0$ for $z \in E$, and

$$
\operatorname{Re}\left\{(1-\alpha) \frac{z f^{\prime}(z)}{f(z)}+\alpha\left[1+\frac{z f^{\prime \prime}(z)}{f^{\prime}(z)}\right]\right\}>0
$$

for $z \in E$ [3], [4]. If $f(z)=z+\sum_{n=2}^{\infty} a_{n} z^{n}$, the coefficient bounds for $\left|a_{2}\right|$ and $\left|a_{3}\right|$ are known [2], [4]; an inequality relating the coefficients $\left|a_{n}\right|$ for $n=2,3, \cdots$ is found in [2]; yet the determination of the coefficient bound for $\left|a_{n}\right|$ has so far been an open problem.

Here we announce the general result for this coefficient problem; the proof will be published elsewhere.

THEOREM. Let $f(z)=z+\sum_{k=2}^{\infty} a_{k} z^{k} \in M(\alpha)$. Let $S(n)$ be the set of all $n$-tuples $\left(r_{1}, r_{2}, \cdots, r_{n}\right)$ of nonnegative integers for which $r_{1}+2 r_{2}+$ $3 r_{3}+\cdots+n r_{n}=n$, and for each such $n$-tuple define $m$ by $r_{1}+r_{2}+\cdots+$ $r_{n}=m$. If $\gamma(\alpha, m)=\alpha(\alpha-1)(\alpha-2) \cdots(\alpha-m)$ with $\gamma(\alpha, 0)=1$, then for $n=1,2, \cdots$

$$
\left|a_{n+1}\right| \leqq \sum \frac{\gamma(\alpha, m-1) c_{1}^{r_{1}} c_{2}^{r_{2}} \cdots c_{n}^{r_{n}}}{r ! r_{2} ! \cdots r_{n} !}
$$

where summation is taken over all n-tuples in $S(n)$, and

$$
c_{n}=\frac{2(2+\alpha)(2+2 \alpha) \cdots[2+(n-1) \alpha]}{n ! \alpha^{n}(1+n \alpha)} .
$$

The bounds in (1) are sharp and for $\alpha>0$ attained by

$$
f(z)=\left[\frac{1}{\alpha} \int_{0}^{z} \zeta^{1 / \alpha-1}(1-\zeta)^{-2 / \alpha} d \zeta\right]^{\alpha} .
$$

AMS (MOS) subject classifications (1970). Primary 30A32, 30A34.

Key words and phrases. $\alpha$-convex univalent functions, coefficient bound. 
For $\alpha=0$, we find from (1) that $\left|a_{n}\right| \leqq n$ for $n=2,3, \cdots$ and the bounds are attained by the function $f(z)=z(1-z)^{-2}$. For $\alpha=1,\left|a_{n}\right| \leqq 1$ for $n=2,3, \cdots$ the bounds being attained by $f(z)=z(1-z)^{-1}$.

The technique used by Goodman in [1] has been employed to get the bounds in (1) in the compact form.

Thus, it is easy to find from (1) that, e.g.,

$$
\begin{aligned}
& \left|a_{2}\right| \leqq 2 /(1+\alpha) \\
& \left|a_{3}\right| \leqq\left(3+8 \alpha+\alpha^{2}\right) /(1+\alpha)^{2}(1+2 \alpha) \\
& \left|a_{4}\right| \leqq \begin{array}{l}
4\left(3+19 \alpha+38 \alpha^{2}+11 \alpha^{3}+\alpha^{4}\right) / 3(1+\alpha)^{3}(1+2 \alpha)(1+3 \alpha) \\
\begin{array}{c}
30+394 \alpha+2024 \alpha^{2}+5284 \alpha^{3}+6386 \alpha^{4}+2638 \alpha^{5} \\
+488 \alpha^{6}+36 \alpha^{7}
\end{array}
\end{array} \\
& \left|a_{5}\right| \leqq \frac{1+\alpha)^{4}(1+2 \alpha)^{2}(1+3 \alpha)(1+4 \alpha)}{6(1+\alpha)}
\end{aligned}
$$

The formula (1) is however readily computable.

It may be noted that $\sup \left|a_{n+1}\right|<\sup \left|a_{n}\right|$ for $\alpha \geqq 2, n=2,3, \cdots$. Also, for a given $n, n=2,3, \cdots, \sup \left|a_{n}\right|$ is a decreasing function of $\alpha, \alpha \geqq 0$.

\section{REFERENCES}

1. A. W. Goodman, Coefficients for the area theorem, Proc. Amer. Math. Soc. 33 (1972), 438-444. MR 45 \#530.

2. P. K. Kulshrestha, Coefficient problems for a class of Mocanu-Bazilevič functions (submitted).

3. P. T. Mocanu, Une propriété de convexité généralisée dans la théorie de la représentation conforme, Mathematica (Cluj) 11 (34) (1969), 127-133. MR 42 \#7881.

4. P. T. Mocanu and M. O. Reade, The order of starlikeness of certain univalent functions (to appear).

Department of Mathematics, Louisiana State University in New Orleans, New ORLEANS, Louisiana 70122 